\title{
Dynamics in Biological Systems as seen by QENS
}

\author{
By A. M. Stadler* \\ Institut Laue-Langevin, 38042 Grenoble, France, and \\ Forschungszentrum Jülich, 52425 Jülich, Germany
}

(Received April 23, 2009; accepted August 20, 2009)

\section{Biology / QENS}

Quasielastic incoherent neutron scattering is a well suited and established experimental method to study protein and water dynamics in the picosecond to nanosecond time- and Ångstrom length-scale. Using deuterium labelling either protein or water motions can be selected and brought into focus. Protein and cell water dynamics were separately studied in red blood cells. A consistent picture of cytoplasmic water and protein dynamics in whole cells is emerging from recent experimental results.

\section{Introduction}

At physiological temperature, in which they are active and fulfil their respective functions, biological macromolecules display a dynamic variety of motions. These occur on various length-scales, from a fraction of one hundredth of a nanometre to several nanometres, and on a wide range of time-scales, from the femtosecond to the second. Proteins are polypeptide chains, which through interactions with their aqueous environment, with themselves, and sometimes with other ligands, fold and assume specific structures that are relevant for biological function. Folding energies are relatively weak, comparable with $\sim k T$, making protein structures very sensitive to temperature and solvent composition. There are two necessary conditions that a protein structure must fulfil: its time-average must be stable under particular physiological conditions; it must display the motions around this time-average that are required for its activity. Water plays an essential (one can safely say vital) role in protein folding, stabilisation and activity. Its physiological state in cells is, therefore, of great interest in the context of

* Corresponding author. E-mail: stadler@ill.fr 
understanding biological function at the molecular level. The dynamical processes are essential for biological function. Measurement and characterisation, in vivo, of such molecular scale dynamics, however, is particularly challenging. Neutron scattering results on molecular dynamics measured in red blood cells $(\mathrm{RBC})$ are reviewed in this article.

Energy-resolved neutron scattering is amongst the few methodologies that have provided experimental data in the area of biological molecular dynamics. In quasielastic neutron scattering (QENS) and elastic incoherent neutron scattering (EINS) experiments, it is the incoherent scattering of $\mathrm{H}$ atoms that is mainly observed and analysed. In the time-scales examined, $\mathrm{H}$ atom motions reflect those of the chemical groups to which they are bound. They are, therefore, excellent indicators of internal and global dynamics in macromolecules, as well as of water dynamics. The scattering cross-section of deuterium $\left(\mathrm{D}\right.$ or $\left.{ }^{2} \mathrm{H}\right)$ is much lower than that of $\mathrm{H}$, so that H-D isotope labelling is a powerful technique to focus on the dynamics of different components of a complex system, e.g. to selectively detect the dynamics of protonated amino-acids in a deuterated protein [1-3].

The cytoplasm of all cells is a crowded environment containing at least $70 \%$ water $[4,5]$. The free distance between macromolecules in the cell is in the order of one nanometre [6]. Similarities and differences in structure and dynamics of cytoplasmic and bulk water have attracted large scientific interest, reviewed by Ball [7]. Water surrounds biological macromolecules, forms their hydration shells and allows them to be functional and active. Because of interactions with the protein surface, hydration shell water displays structural and dynamic differences with bulk water, reviewed by Bellissent-Funel [8]. The question then is: does the cytoplasmic environment modify the properties of water (compared with the bulk phase) beyond the immediate hydration shells of macromolecules? It is an important question because most biochemical studies are performed in fractionated systems in dilute solution, with the assumption that the observations are significant for the corresponding cellular process. In vitro EINS work on proteins in membranes [9, 10], in solution and in hydrated powder samples [2, 11, 12] has established that protein dynamics is strongly hydration dependent. It was of significant interest, therefore, to measure protein dynamics in vivo.

Cells are highly complex systems. Eukaryotic cells consist of several organelles (nucleus, mitochondria, golgi complex, etc.). They contain thousands of different proteins and large amounts of DNA, RNA and ribosomes. Many proteins are involved in large macromolecular complexes and assemblies. Cells are far from being static, e.g. during mitosis they can change their composition. Prokaryotic cells, such as E. coli, are somehow simpler as they do not have any organelles. They still contain many different proteins, including large fractions of DNA and RNA. Average properties of these cells are therefore difficult to attribute precisely to a certain component. $\mathrm{RBC}$ on the other side are highly specialised and very simple eukaryotic cells. RBC do not contain any organelles or DNA/RNA and their main constituent is hemoglobin $(\mathrm{Hb})$ at $92 \%$ of dry 
weight. The concentration of $\mathrm{Hb}$ in $\mathrm{RBC}$ is $330 \mathrm{mg} / \mathrm{ml}$ [6], which corresponds to a volume fraction of 0.25 . Using the above mentioned techniques of hydrogen/ deuterium labelling, average dynamics of whole cells as measured with incoherent neutron scattering can be attributed to either macromolecular $\mathrm{Hb}$ or $\mathrm{H}_{2} \mathrm{O}$ dynamics. $\mathrm{Hb}$ is a tetramer with a molecular mass of $64 \mathrm{kDa}$. It consists of two so called $\alpha$ - and two $\beta$-chains, each of them have 141 and 146 amino acids, respectively [13]. Every chain carries one heme-group which can reversibly bind oxygen and other diatomic molecules. Binding of oxygen to deoxygenated $\mathrm{Hb}$ results in a change of the structure of $\mathrm{Hb}$ [14]. The fully oxygenated form is called R-state (relaxed state), whereas the deoxygenated conformation of $\mathrm{Hb}$ is termed T-state (tensed state). Binding of $\mathrm{O}_{2}$ to $\mathrm{Hb}$ is cooperative and $\mathrm{Hb}$ is a prototype of a protein which exhibits allosteric regulation [15].

\section{Experimental}

$\mathrm{Hb}$ dynamics in powders, solutions and RBC were measured by EINS on IN13 with an energy resolution of $\Delta E=8 \mu \mathrm{eV}$ full-width at half-maximum (FWHM), and by QENS on TOFTOF (FWHM $\Delta E=100 \mu \mathrm{eV}$ ) and FOCUS (FWHM $\Delta E$ $\sim 50 \mu \mathrm{eV})[11,16]$.

At the energy resolutions of the time-of-flight spectrometers global macromolecular diffusion is strongly suppressed or even absent in the concentrated $\mathrm{Hb}$ solution and powder samples, respectively. The scattering function $S(q, \omega)$ of $\mathrm{Hb}$ internal dynamics was therefore approximated as

$$
S(q, \omega)=e^{-<x^{2}>q^{2}} \cdot\left[A_{0}(q) \times \delta(\omega)+\frac{\left(1-A_{0}(q)\right)}{\pi} \times \frac{\Gamma_{I}(q)}{\omega^{2}+\Gamma_{I}(q)^{2}}\right],
$$

where $A_{0}(q)$ is the Elastic Incoherent Structure Factor (EISF), $<x^{2}>$ is proportional to mean square displacements of fast vibrational motions and $q$ is the modulus of the scattering vector. The observed average protein dynamics were detected with one Lorentzian with the half-widths at half-maximum (HWHM) $\Gamma_{I}(q)$. In the RBC sample, global macromolecular diffusion contributes to the measured intensities and the scattering function then reads

$$
\begin{aligned}
& S(q, \omega)= \\
& e^{-\left\langle x^{2}\right\rangle q^{2}} \cdot\left[\frac{A_{0}(q)}{\pi} \cdot \frac{\Gamma_{G}(q)}{\omega^{2}+\Gamma_{G}(q)^{2}}+\frac{1-A_{0}(q)}{\pi} \cdot \frac{\Gamma_{G}(q)+\Gamma_{I}(q)}{\omega^{2}+\left[\Gamma_{G}(q)+\Gamma_{I}(q)\right]^{2}}\right]
\end{aligned}
$$

with an additional Lorentzian with the HWHM $\Gamma_{G}(q)$ for global Hb diffusion. The scattering functions plus linear background were convoluted with the instrumental energy resolution and fitted to the measured data.

Total mean square displacements $\left\langle u^{2}\right\rangle$, MSD, were calculated from the measured elastic intensities $I(q)$ on IN13 according to $\left.<u^{2}\right\rangle=\frac{-6 \cdot \Delta \ln I(q)}{\Delta q^{2}}$ for $q^{2} \rightarrow 0$ and plotted against temperature. 
QENS data from neutron time-of-flight and backscattering spectrometers with various energy resolution values $\Delta E$ were combined for the experiments on cell water dynamics. The corresponding time-scales covered the range from that of bulk water to that of reduced mobility interfacial water motions. RBC water was measured on IRIS (FWHM $\Delta E=17 \mu \mathrm{eV}$ ), FOCUS (FWHM $\Delta E \sim 50 \mu \mathrm{eV}$ ) at PSI and TOFTOF (FWHM $\Delta E=100 \mu \mathrm{eV}$ ) at FRM-II [17].

The scattering function $S(q, \omega)$ for translational and rotational diffusion of water was approximated as

$$
S(q, \omega)=e^{-\left\langle x^{2}\right\rangle \cdot q^{2}}\left\{\frac{A_{1}(q)}{\pi} \cdot \frac{\Gamma_{T}(q)}{\omega^{2}+\Gamma_{T}(q)^{2}}+\frac{A_{2}(q)}{\pi} \cdot \frac{\Gamma_{T}(q)+\Gamma_{R}(q)}{\omega^{2}+\left[\Gamma_{T}(q)+\Gamma_{R}(q)\right]^{2}}\right\},
$$

the first Lorentzian in the curly brackets represents translational diffusion, the second Lorentzian combines translational and rotational diffusion with the HWHM $\Gamma_{T}(q)$ and $\Gamma_{R}(q)$, respectively. The scattering function was convoluted with the instrumental resolution function and fitted to the measured spectra $S_{\exp }(q, \omega)$ according to

$$
S_{\exp }(q, \omega)=\left[e^{-\left\langle x^{2}\right\rangle q^{2}} \cdot A_{0}(q) \cdot \delta(\omega)+S(q, \omega)+B\right] \otimes S_{\text {res }}(q, \omega)
$$

where $A_{0}(q)$ is the EISF of slow hydrogen dynamics that appear localized within the instrumental energy resolution and $B$ is linear background. TOFTOF and FOCUS data were interpreted according to equations (3) and (4), whereas for IRIS it was sufficient to describe the data with one Lorentzian for translational dynamics.

Translational diffusion coefficients, and jump model parameters were calculated from the $q^{2}$ dependence of the $\Gamma_{T}(q)$, where $q$ is the scattering vector modulus.

\section{Protein dynamics in red blood cells}

In a first set of experiments we studied the dynamics of $\mathrm{Hb}$ in $\mathrm{RBC}$ with QENS [16]. $\mathrm{RBC}$ were repeatedly washed with saline $\mathrm{D}_{2} \mathrm{O}$ buffer to reduce the scattering contribution of water and to focus on protein dynamics. In our approach we could separate global protein diffusion from internal $\mathrm{Hb}$ motions. We complemented our neutron experiments with dynamic light scattering measurements to obtain the diffusion coefficient of $\mathrm{Hb}$ in dilute solution.

Doster and Longeville studied global $\mathrm{Hb}$ diffusion in $\mathrm{RBC}$ using neutron spin-echo spectroscopy [18]. The authors compared the obtained long-time limit of the diffusion coefficient with predicted values of hydrodynamic theory of non-charged colloids with hard-spheres interactions. It was demonstrated that quantitative agreement between measurement and theory can be reached when part of the hydration shell is assumed to be attached to the surface of $\mathrm{Hb}$ and to move in a joint way. In our study using neutron time-of-flight spectroscopy, we 


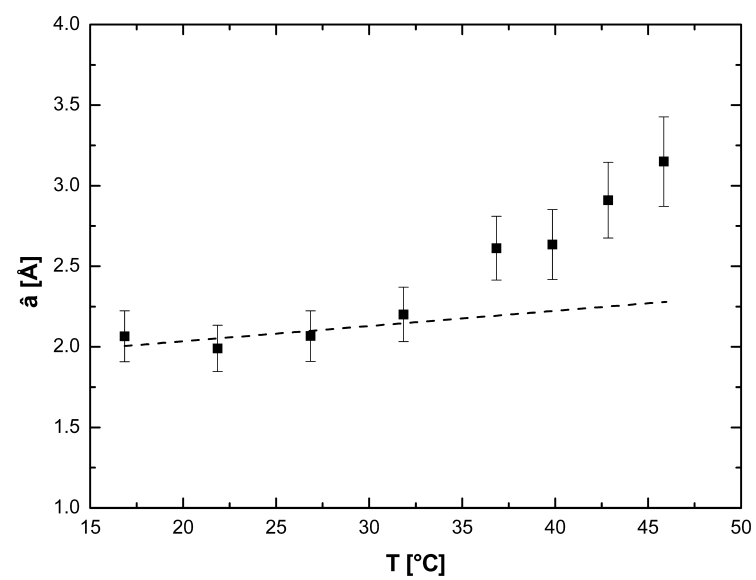

Fig. 1. Internal $\mathrm{Hb}$ dynamics measured in whole RBC [16]: Temperature dependence of the average radius $\hat{a}$ of internal $\mathrm{Hb}$ dynamics. The dashed line is a linear fit to the values between $16.9^{\circ} \mathrm{C}$ and $31.9^{\circ} \mathrm{C}$ and serves only as a guide for the eye. The increase of flexibility above $36.9^{\circ} \mathrm{C}$ was interpreted due to partial unfolding of $\mathrm{Hb}$ at body temperature.

also compared the measured global diffusion coefficients of $\mathrm{Hb}$ to theoretical predictions for concentrated suspensions of non-charged hard-sphere colloids. The values of the diffusion coefficients in the cells were found to be in the same order as it is expected for short-time self-diffusion.

Conclusions about internal $\mathrm{Hb}$ dynamics can be drawn from the amplitudes of the EISF and the half-widths of the quasielastic broadening. Doster and Settles determined the displacement distribution of hydrated myoglobin with neutron scattering [19]. The authors found that a protein is characterised by a rather broad bimodal displacement distribution which consists of a vibrational part and a component that was attributed to rotational jumps of side-chains [19]. In an alternative approach, measured EISF of internal protein dynamics were interpreted with a model for 'diffusion in a sphere' [20, 21]. In this approach, the observed picosecond protein motions are attributed to the diffusion of side-chains within a restricted spherical volume. It was shown that it is more appropriate to use a distribution of sphere radii combined with the 'diffusion in a sphere' model to describe measured EISF more accurately [22], with a Gaussian distribution of sphere radii as the simplest approximation. In our study on $\mathrm{Hb}$ dynamics in $\mathrm{RBC}$, the EISF was fitted using a model for 'diffusion in a sphere' with a Gaussian distribution of sphere radii to take into account for the dynamic heterogeneity of protein dynamics. The detected dynamics in the order of some picoseconds were attributed to localized diffusive motions of amino acid side-chains.

The mean value $\hat{a}$ of the Gaussian distribution is plotted as a function of temperature in Figure 1. The error bars are rather large but up to $31.9^{\circ} \mathrm{C}$ the mean value increases linearly with temperature. Above $36.9^{\circ} \mathrm{C}$, there is a signifi- 


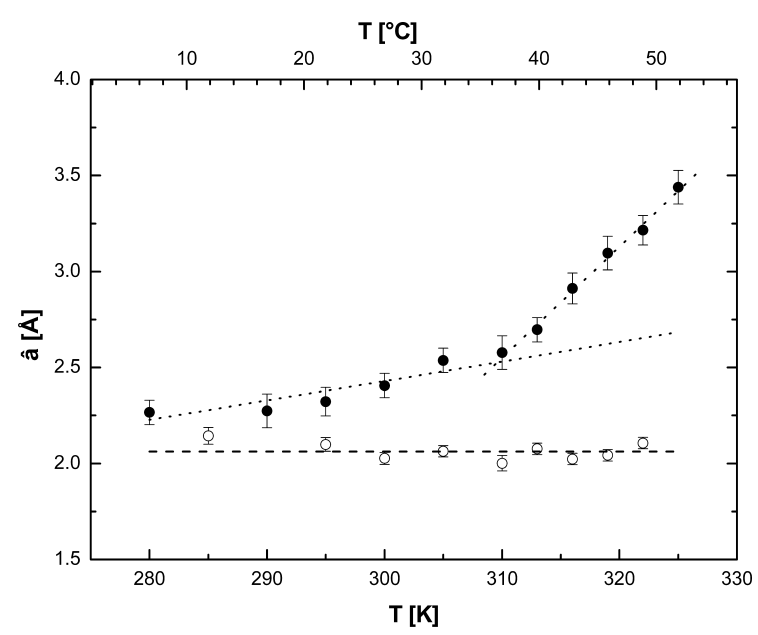

Fig. 2. Mean value $\hat{a}$ of the sphere radii of $\mathrm{Hb}$ as a function of temperature [11]: $\mathrm{Hb}$ in solution (filled circles) and hydrated Hb powder (empty circles). The dashed and dotted lines are linear fits and serve as a guide for the eye.

cant increase in $\hat{a}$ which indicates an increase in the flexibility of the protein. Complementary studies on dilute $\mathrm{Hb}$ solutions using circular dichroism [23, 24] and micropipette aspiration experiments on single $\operatorname{RBC}[25,26]$ reported that $\mathrm{Hb}$ senses body temperature and exhibits 'partial unfolding' of its structure above body temperature. Our study on internal $\mathrm{Hb}$ dynamics is in agreement with these results and would further indicate that the flexibility of $\mathrm{Hb}$ is larger in the "partial unfolded' than in the folded state.

\section{Hydration dependence of hemoglobin dynamics}

A subsequent set of experiments was designed to test whether or not the body temperature effect existed in $\mathrm{Hb}$ outside RBC [11]. Hb dynamics was measured in different samples from solution $\left(\sim 570 \mathrm{mg} / \mathrm{ml} \mathrm{Hb}\right.$ in $\mathrm{D}_{2} \mathrm{O}$ buffer $)$ to hydrated powder $\left(0.4 \mathrm{~g} \mathrm{D}_{2} \mathrm{O} / \mathrm{g} \mathrm{Hb}\right)$. Our goal in the study was to investigate in detail with neutron scattering, how motions of human $\mathrm{Hb}$ depend on hydration.

To be comparable with the study of Hb dynamics in RBC, we used the same 'diffusion in a sphere' model with a Gaussian distribution of radii to interpret the measured EISF. The average sphere radii $\hat{a}$ of $\mathrm{Hb}$ in concentrated solution and as hydrated powder are presented in Figure 2. A break in the temperature dependence of the average sphere radii was detected close to human body temperature in the concentrated $\mathrm{Hb}$ solution but not in the hydrated powder sample.

Furthermore, we investigated the behavior of the quasielastic broadening of both samples. Measured half-widths of the Lorentzians are presented in Fig- 

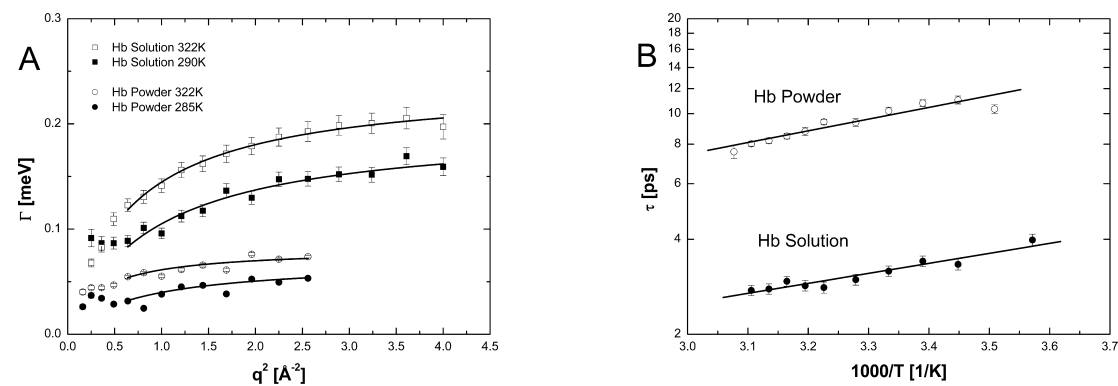

Fig. 3. (A) Half-widths at half-maximum $\Gamma$ of the Lorentzian for internal protein dynamics as a function of the scattering vector at different temperatures of $\mathrm{Hb}$ in solution and as hydrated powder [11]. The solid lines are fits according to a jump-diffusion model in the $q^{2}$-range from 0.64 to $4.0 \AA^{-2}$ and from 0.64 to $2.56 \AA^{-2}$. (B) Residence time $\tau$ of $\mathrm{Hb}$ in solution (filled circles) and hydrated $\mathrm{Hb}$ powder (empty circles). The straight lines indicate fits according to the Arrhenius law. Activation energies of $E_{a}=1.45 \pm 0.18 \mathrm{kcal} / \mathrm{mol}$ for $\mathrm{Hb}$ in solution, and $E_{a}=1.70 \pm 0.12 \mathrm{kcal} / \mathrm{mol}$ for hydrated $\mathrm{Hb}$ powder were obtained.

ure 3(A). The line-widths approach a plateau at high $q^{2}$-values which is a typical feature of jump-diffusion. At small scattering vectors, the HWHM do not intercept zero as it would be expected for free diffusion, but they tend towards a constant value which is a sign for motion in confinement. At large scattering values, a jump-diffusion model $\Gamma=\frac{D q^{2}}{1+D q^{2} \tau}$, with the jump-diffusion coefficient $D$ and the residence time between jumps $\tau$, was used to describe the HWHM. The values of the residence times are given in Figure 3(B).

Our experiments revealed that increasing hydration of $\mathrm{Hb}$ has a strong influence on the rates of diffusive motions. The obtained activation energy $E_{a}$ of the residence times raises from $1.45 \pm 0.18 \mathrm{kcal} / \mathrm{mol}$ for $\mathrm{Hb}$ solution to $1.70 \pm 0.12 \mathrm{kcal} / \mathrm{mol}$ for hydrated $\mathrm{Hb}$ powder. We concluded that an increase of hydration from a single layer to nearly three layers enhances the rates of diffusive motions. The supplementary amount of water decreases the activation energy barriers between diffusive jumps and thus facilitates protein dynamics, as shown with QENS. In the picture of Fraunfelder and co-workers, proteins exist in many slightly different conformational substates which are separated by activation energy barriers [27]. The sampling of the conformational substates contributes to the entropic stabilization of proteins. Lower activation energy barriers allow an increased sampling rate of the conformational substates. The other term that determines protein stability is the enthalpic contribution which is determined by the macromolecular force field [28].

Additionally, we measured mean square displacements $\left\langle u^{2}\right\rangle$ as a function of temperature of the hydrated $\mathrm{Hb}$ powder sample on the backscattering spectrometer IN13 at ILL to confirm the presence of the dynamical transition at around 180-200 K [29] in the sample. Typical measured intensities $I(q)$ are 

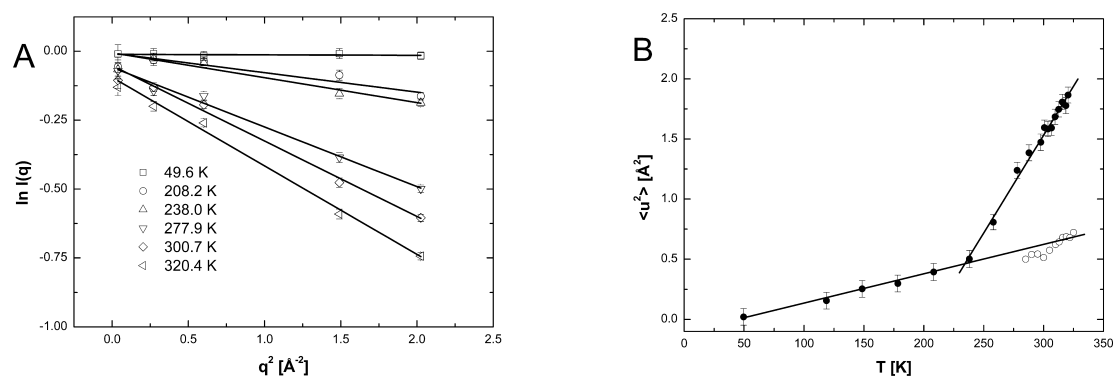

Fig. 4. (A) Elastic intensities of hydrated $\mathrm{Hb}$ powder measured on IN13. Straight lines indicate fits to extract the $\left\langle u^{2}\right\rangle$. (B) Mean square displacements $\left\langle u^{2}\right\rangle$ obtained from elastic scans on IN13 (filled circles) and mean square displacements of vibrational motions $\left\langle u^{2}\right\rangle_{v i b}$ determined with QENS on FOCUS (empty circles) [11].

shown in Figure 4 (A) and the obtained $\left\langle u^{2}\right\rangle$ are given in Figure 4(B). The $<u^{2}>$ demonstrate that the dynamical transition is present in the hydrated powder sample. Mean square displacements of fast vibrational motions of hydrated $\mathrm{Hb}$ powder measured on FOCUS are also given in Figure 4 (B). To allow a direct comparision between the $\left\langle u^{2}\right\rangle$ and $\left\langle x^{2}\right\rangle$ values, the vibrational motions detected on FOCUS are scaled and given as $\left\langle u^{2}\right\rangle_{\text {vib }}=6 \cdot\left\langle x^{2}\right\rangle$ in the figure. At low temperatures only solid-like vibrational motions are detected in the $\left\langle u^{2}\right\rangle$; at temperatures above the dynamic transition temperature protein specific quasidiffusive motions are activated and contribute to the measured $\left.<u^{2}\right\rangle$ [30]. From QENS experiments vibrational and diffusive motions can be separated. As expected, the obtained vibrational motions $\left\langle u^{2}\right\rangle_{v i b}$ are in good agreement with linear extrapolation of low temperature $\left\langle u^{2}\right\rangle$ obtained from the EINS measurement.

In the previous study of $\mathrm{Hb}$ dynamics in $\mathrm{RBC}$, the break at $310 \mathrm{~K}$ in the sphere radii was interpreted as partial unfolding of $\mathrm{Hb}$ at human body temperature [16]. The same interpretation is valid for the results of $\mathrm{Hb}$ in concentrated solution. In this sense, the partially unfolded state of $\mathrm{Hb}$ solution at temperatures above $310 \mathrm{~K}$ has got higher flexibility than the low temperature state, as the Gaussian distribution of radii is shifted to larger volumes. The fact that partial unfolding and the consequent changes of dynamics do not occur in hydrated powder implies a crucial role of hydration in this process. It was previously suggested that solvent accessible amino acid side-chains might be responsible for $\mathrm{Hb}$ partial unfolding $[31,32]$ which leads to $\mathrm{Hb}$ aggregation at temperatures above body temperature [25]. The observed changes in the dynamics of amino acid side-chains at body temperature might be responsible for an increase in surface hydrophobicity that facilitates protein association and aggregation [24, 33]. The experimental facts presented in this work justify this view. Diffusive motion rates of side-chains are strongly suppressed in hydrated $\mathrm{Hb}$ powder as compared to $\mathrm{Hb}$ in concentrated solution. We concluded before that protein dy- 
namics and changes in the complex macromolecular force field might be responsible for small structural changes and pronounced protein aggregation above body temperature [33]. The molecular properties of $\mathrm{Hb}$ therefore could determine in this sense the macroscopic properties of whole RBC [34]. We identified a fast process in the order of some picoseconds that might be responsible for the observed changes in $\mathrm{Hb}$ dynamics at body temperature. A sufficient level of hydration beyond one surface layer is crucial for the activation of this fast process.

\section{Water dynamics in cells}

A 2006 book edited by Pollack et al. states already in the preface '... practically all cell water is interfacial' [35]. It was suggested that the cell interior is a gel or colloidal-like structure in which confined water is interfacial with properties that are significantly different from those of pure water. The claim about water dynamics was derived from indirect macroscopic experimental observations and remains controversial, reviewed by Ball [7]. It is interesting to note that a recent QENS study has shown that water diffusion in clay gels was similar to bulk water in the A-ps length- and time-scale [36]. The apparent diffusion coefficient of water in various biological tissues, including the brain, has been measured by NMR. On a micrometric scale, water diffusion appeared to be reduced by a factor of between 2 to 10 compared to pure water, review by Le Bihan [37]. In contrast, very little was known about cell water dynamics on sub-micrometric and sub-nanosecond scales.

Water is essential to life and a major scientific interest lies in a detailed understanding of how it interacts with biological macromolecules in cells. The cellular environment is extremely crowded with macromolecular concentrations up to $400 \mathrm{mg} / \mathrm{ml}$ [38]. Distances between macromolecules are in the order of $1 \mathrm{~nm}$, which corresponds to only few layers of water. Water that is in close contact with hydrophilic or hydrophobic protein surfaces [39] or which is trapped in surface cavities [40] is shown to have significantly different behavior than bulk water. Recent studies point out that a major fraction of water in cells and bacteria shows bulk like dynamics [41, 42]. We measured QENS of $\mathrm{H}_{2} \mathrm{O}$ in $\mathrm{RBC}$, in vivo. The data revealed two populations of water in RBC: a major fraction of $\sim 90 \%$ which has dynamical properties similar to those of bulk water (time scale $\sim$ ps) and a minor fraction of $\sim 10 \%$ which is interpreted as bound hydration water with significantly slower dynamics (time scale $\sim 40 \mathrm{ps}$ ).

Cell water dynamics was measured on the instruments IRIS at ISIS, FOCUS at PSI and TOFTOF at FRM-II. The neutron spectrometers are characterized by their specific energy resolution and scattering vector range, which determine the observable time and lengths scales of hydrogen motions. The energy resolutions of the instruments IRIS, FOCUS and TOFTOF were $17 \mu \mathrm{eV}, 50 \mu \mathrm{eV}$ and $100 \mu \mathrm{eV}$, respectively, corresponding to observable motions in the time scales in the order of $40 \mathrm{ps}, 13 \mathrm{ps}$ and 7 ps. As fully deuterated RBC are not available, 

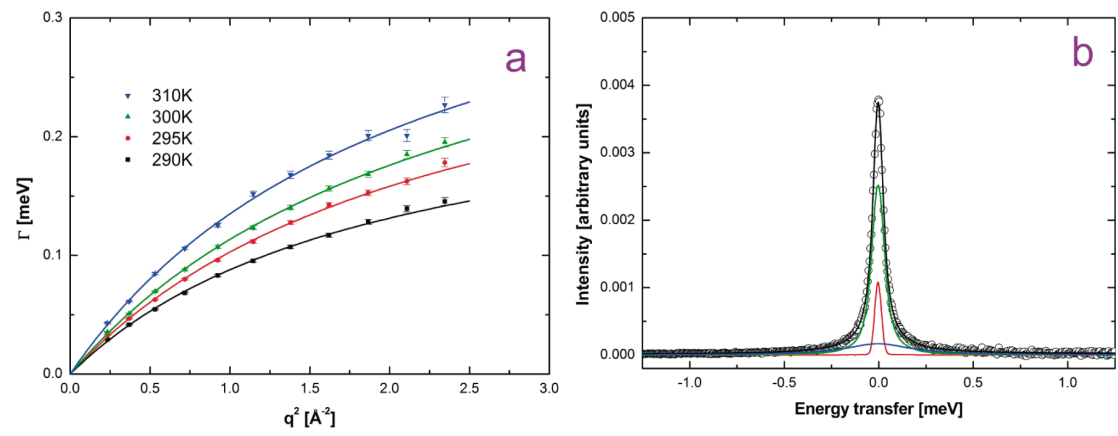

Fig. 5. a) Half-widths at half-maximum of the translational diffusion process of cytoplasmic $\mathrm{H}_{2} \mathrm{O}$ in RBC measured on IRIS at different temperatures. Solid lines represent fits according to a jump-diffusion model. b) QENS spectrum on FOCUS of cytoplasmic $\mathrm{H}_{2} \mathrm{O}$ in $\mathrm{RBC}$ at $290 \mathrm{~K}$ and $q=0.55 \AA^{-1}$. Symbols show measured data, the black line presents the result of the fit. The components correspond to the immobile fraction (red line), the narrow Lorentzian (green line) and the broad Lorentzian (blue line) [17].

natural abundance $\mathrm{RBC}$ in $\mathrm{H}_{2} \mathrm{O}$ buffer and natural abundance $\mathrm{RBC}$ in $\mathrm{D}_{2} \mathrm{O}$ buffer were measured separately. To focus on $\mathrm{H}_{2} \mathrm{O}$ dynamics, the measured intensities of the $\mathrm{RBC}$ in $\mathrm{D}_{2} \mathrm{O}$ buffer sample were subtracted from the $\mathrm{RBC}$ in $\mathrm{H}_{2} \mathrm{O}$ buffer sample.

Human RBC samples were prepared in an identical way in $\mathrm{H}_{2} \mathrm{O}$ and $\mathrm{D}_{2} \mathrm{O}$ buffer and concentrated by centrifugation. The water content in the samples was determined by drying and weighing aliquots. The extracellular water content was found to be less than $10 \%$ of the total water content assuming a cellular $\mathrm{Hb}$ concentration of $330 \mathrm{mg} / \mathrm{ml}$. We estimate that the incoherent cross section of the intracellular $\mathrm{H}_{2} \mathrm{O}$ is more than three times that of the macromolecules. In $\mathrm{D}_{2} \mathrm{O}$, the incoherent cross section of water is $\sim 10 \%$ of the macromolecules [16] when the exchangeable protons of $\mathrm{Hb}$ are also considered. The difference intensities are therefore dominated by the water signal, and we additionally assume that membrane and macromolecular dynamics are similar in $\mathrm{H}_{2} \mathrm{O}$ and $\mathrm{D}_{2} \mathrm{O}$ buffer.

The line-widths of the translational component $\Gamma(q)$ of cell water measured on the instrument IRIS are shown with fits according to a jump-diffusion model $\Gamma(q)=\frac{D q^{2}}{1+D q^{2} \tau}$ in Figure 5 a). In Figure 5 b) a measured spectrum on FOCUS together with the fitted components is presented.

The obtained translational diffusion coefficients $D$ of $\mathrm{H}_{2} \mathrm{O}$ buffer and cytoplasmic $\mathrm{H}_{2} \mathrm{O}$ as a function of temperature are presented in Figure 6 a). The data demonstrate that the translational diffusion coefficient of cellular water is nearly identical to $\mathrm{H}_{2} \mathrm{O}$ buffer on all spectrometers. The dashed line indicates normal temperature behavior of $\mathrm{H}_{2} \mathrm{O}$ buffer following an Arrhenius law and serves as reference. However, residence times were found to be larger in intracellular than 

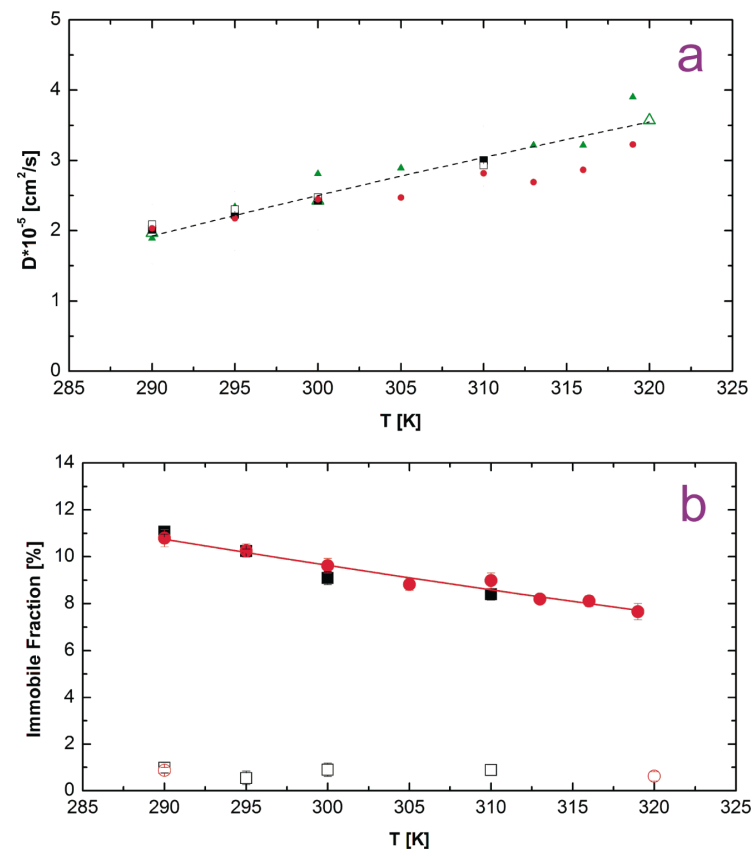

Fig. 6. a) Translational diffusion coefficient $D$ of $\mathrm{H}_{2} \mathrm{O}$ in $\mathrm{RBC}$ measured on the instruments IRIS (filled black squares), FOCUS (filled red circles) and TOFTOF (filled green triangles); $\mathrm{H}_{2} \mathrm{O}$ buffer was measured on the instruments IRIS (empty black squares) and TOFTOF (empty green triangles). The dashed line indicates normal temperature behaviour of $\mathrm{H}_{2} \mathrm{O}$ buffer [17]. b) Percentage of immobile fraction of $\mathrm{H}_{2} \mathrm{O}$ in RBC measured on IRIS at $q=0.61 \AA^{-1}$ (filled black squares) and FOCUS at $q=0.55 \AA^{-1}$ (filled red circles) are compared to values of $\mathrm{H}_{2} \mathrm{O}$ buffer measured on IRIS (empty black squares) and FOCUS (empty red circles). The straight red line represents Arrhenius behaviour.

in bulk water. The results indicate that translational diffusion of water in RBC behaves similarly to bulk water.

Furthermore, we identified a significant fraction of immobile water in RBC that is absent in $\mathrm{H}_{2} \mathrm{O}$ buffer. The immobile water fraction was determined by the amplitude of the elastic peak divided by the integrated total intensity. Experimental data is presented in Figure $6 \mathrm{~b}$ ) for the smallest accessible scattering vector of $q \sim 0.6 \AA^{-1}$ which reports on all movements up to around $10 \AA$ real space distance. On this length scale confining effects of protein surface cavities or boundaries on water become observable. The obtained immobile fraction of cellular water varies between $11 \%$ and $8 \%$ in the investigated temperature range. The uncertainty due to background subtraction was estimated to be below $2 \%$. The immobile fraction was attributed to water which is dynamically bound on the surface of $\mathrm{Hb}$.

Hydrodynamic experiments on $\mathrm{Hb}$ required the assumption of a bound water coverage of around $50 \%$ of the first hydration layer around the protein, in order 
to predict quantitatively the measured parameters $[43,44]$. From the known $\mathrm{Hb}$ concentration inside RBC, we calculated that $\sim 50 \%$ of the hydration layers does in fact correspond to about $10 \%$ of the total cytoplasmic water, in agreement with the analysis of the elastic intensities.

The QENS results on RBC established firmly that water diffusion, beyond the narrow hydration shells around macromolecules, is neither confined nor significantly slowed down compared to pure water. Contrary to the widespread belief that water is "tamed" by macromolecular confinement, cell water diffusion beyond the hydration shell is similar to that of pure water at physiological temperature. The same conclusion has been reached using NMR spectroscopy by Persson and Halle [42]. The authors have examined the rotational spin relaxation rate of $E$. coli water, over a wide time range from the millisecond to the picosecond time-scale, and found that about $85 \%$ of $E$. coli water presented rotational relaxation times similar to those of pure water. The remaining $15 \%$ of cell water in $E$. coli was interpreted to $\mathrm{H}_{2} \mathrm{O}$ molecules with slow dynamics which are in the hydration shell of biomolecules. The study of Persson and Halle therefore supports our results and interpretation of $\mathrm{H}_{2} \mathrm{O}$ dynamics in $\mathrm{RBC}$.

\section{Summary}

In conclusion, the following results were obtained from the in vivo QENS and EINS measurements of biologically significant molecular dynamics: Beyond the hydration shell, which account for about $10 \%$ of the total water content in cells corresponding to half a layer of water around macromolecules, cytoplasmic water flows as freely as bulk water in RBC in the $\AA$-ps space-time window [17, 45]. $\mathrm{Hb}$ dynamics in $\mathrm{RBC}$ was found to be correlated to body temperature [16]. Comparison of data from $\mathrm{RBC}$, from concentrated $\mathrm{Hb}$ solution and hydrated $\mathrm{Hb}$ powder samples indicated the great sensitivity of protein dynamics to hydration conditions [11]. In particular, it appeared that even fully hydrated powders do not display the same range of motions as $\mathrm{Hb}$ in the cell and in concentrated solution.

\section{Acknowledgement}

The author thanks Drs. Giuseppe Zaccai and Marion Jasnin for invaluable discussion and critical reading of the manuscript.

\section{References}

1. K. Wood, et al., Dynamical heterogeneity of specific amino acids in bacteriorhodopsin. J. Mol. Biol. 380(3) (2008) 581-91.

2. K. Wood, et al., Hydration dependence of active core fluctuations in bacteriorhodopsin. Biophys. J. 95(1) (2008) 194-202. 
3. V. Reat, et al., Dynamics of different functional parts of bacteriorhodopsin: $H-2 H$ labeling and neutron scattering. Proc Natl Acad Sci USA95(9) (1998) 4970-4975.

4. D. Hall and A. P. Minton, Macromolecular crowding: qualitative and semiquantitative successes, quantitative challenges. Biochim Biophys Acta 1649(2) (2003) 12739.

5. A. P. Minton, The influence of macromolecular crowding and macromolecular confinement on biochemical reactions in physiological media. J. Biol. Chem. 276(14) (2001) 10577-80.

6. S. Krueger and R. Nossal, SANS studies of interacting hemoglobin in intact erythrocytes. Biophys. J. 53(1) (1988) 97-105.

7. P. Ball, Water as an active constituent in cell biology. Chem. Rev. 108(1) (2008) 74-108.

8. M. C. Bellissent-Funel, Structure of confined water. Journal of Physics-Condensed Matter 13(41) (2001) 9165-9177.

9. M. Ferrand, et al., Thermal motions and function of bacteriorhodopsin in purple membranes: effects of temperature and hydration studied by neutron scattering. Proc. Natl. Acad. Sci. USA90(20) (1993) 9668-72.

10. K. Wood, et al., Coupling of protein and hydration-water dynamics in biological membranes. Proc. Natl. Acad. Sci. USA104(46) (2007) 18049-54.

11. A. M. Stadler, et al., From powder to solution: hydration dependence of human hemoglobin dynamics correlated to body temperature. Biophys. J. 96(12) (2009) 5073-81.

12. E. Fabiani, et al., Dynamics of apomyoglobin in the alpha-to-beta transition and of partially unfolded aggregated protein. Eur. Biophys. J. 38(2) (2009) 237-44.

13. M. F. Perutz, et al., Stereochemistry of Cooperative Mechanisms in Hemoglobin. Accounts of Chemical Research 20(9) (1987) 309-321.

14. M. F. Perutz, Stereochemistry of Cooperative Effects in Haemoglobin: Haem-Haem Interaction and the Problem of Allostery. Nature 228(5273) (1970) 726-734.

15. J. Monod, J. Wyman, and J.-P. Changeux, On the Nature of Allosteric Transitions: A Plausible Model. J. Mol. Biol. 12(1) (1965) 88-118.

16. A. M. Stadler, et al., Hemoglobin dynamics in red blood cells: correlation to body temperature. Biophys. J. 95(11) (2008) 5449-61.

17. A. M. Stadler, et al., Cytoplasmic water and hydration layer dynamics in human red blood cells. J. Am. Chem. Soc. 130(50) (2008) 16852-3.

18. W. Doster and S. Longeville, Microscopic diffusion and hydrodynamic interactions of hemoglobin in red blood cells. Biophys. J. 93(4) (2007) 1360-8.

19. W. Doster and M. Settles, Protein-water displacement distributions. Biochim. Biophys. Acta 1749(2) (2005) 173-86.

20. J. Fitter, A Measure of Conformational Entropy Change during Thermal Protein Unfolding Using Neutron Spectroscopy. Biophys. J. 84(6) (2003) 3924-3930.

21. J. Fitter and J. Heberle, Structural equilibrium fluctuations in mesophilic and thermophilic alpha-amylase. Biophys. J. 79(3) (2000) 1629-36.

22. J. Perez, J. M. Zanotti, and D. Durand, Evolution of the internal dynamics of two globular proteins from dry powder to solution. Biophys. J. 77(1) (1999) 454-69.

23. G. M. Artmann, et al., Circular dichroism spectra of human hemoglobin reveal a reversible structural transition at body temperature. Eur. Biophys. J. 33(6) (2004) 490-6.

24. I. Digel, et al., Body temperature-related structural transitions of monotremal and human hemoglobin. Biophys. J. 91(8) (2006) 3014-21.

25. G. M. Artmann, et al., Hemoglobin senses body temperature. Eur. Biophys. J. 38(5) (2009) 589-600.

26. G. M. Artmann, et al., Temperature transitions of protein properties in human red blood cells. Biophys. J. 75(6) (1998) 3179-83. 
27. H. Frauenfelder, S. G. Sligar, and P. G. Wolynes, The energy landscapes and motions of proteins. Science 254(5038) (1991) 1598-603.

28. G. Zaccai, How soft is a protein? A protein dynamics force constant measured by neutron scattering. Science 288(5471) (2000) 1604-7.

29. W. Doster, S. Cusack, and W. Petry, Dynamical transition of myoglobin revealed by inelastic neutron scattering. Nature 337(6209) (1989) 754-6.

30. F. Parak, E. W. Knapp, and D. Kucheida, Protein dynamics. Mossbauer spectroscopy on deoxymyoglobin crystals. J. Mol. Biol. 161(1) (1982) 177-94.

31. I. Digel, et al., Body temperature-related structural transitions of monotremal and human hemoglobin. Biophys. J. 91(8) (2006) 3014-21.

32. K. F. Zerlin, et al., Structural transition temperature of hemoglobins correlates with species' body temperature. Eur. Biophys. J. 37(1) (2007) 1-10.

33. A.M. Stadler, et al., Hemoglobin Dynamics in Red Blood Cells: Correlation to Body Temperature. Biophys. J. 95 (2008) 5449-5461.

34. G. M. Artmann, et al., Temperature transitions of protein properties in human red blood cells. Biophys. J. 75(6) (1998) 3179-83.

35. G. H. Pollack, I. L. Cameron, and D. N. Wheatley, Water and the Cell. Dordrecht, The Netherlands: Springer (2006).

36. T. Seydel, et al., Unaffected microscopic dynamics of macroscopically arrested water in dilute clay gels. Phys Rev E 78(6) (2008) 061403.

37. D. Le Bihan, The 'wet mind': water and functional neuroimaging. Phys. Med. Biol. 52(7) (2007) R57-R90.

38. R. J. Ellis and A. P. Minton, Cell biology: join the crowd. Nature 425(6953) (2003) $27-8$.

39. D. Russo, et al., Molecular view of water dynamics near model peptides. J. Phys. Chem. B. 109(26) (2005) 12966-75.

40. V. A. Makarov, et al., Residence times of water molecules in the hydration sites of myoglobin. Biophys. J. 79(6) (2000) 2966-74.

41. M. Jasnin, et al., Down to atomic-scale intracellular water dynamics. EMBO Rep 9(6) (2008) 543-7.

42. E. Persson and B. Halle, Cell water dynamics on multiple time scales. Proc. Natl. Acad. Sci. USA105(17) (2008) 6266-71.

43. J. Garcia de la Torre, Hydration from hydrodynamics. General considerations and applications of bead modelling to globular proteins. Biophys. Chem. 93(2-3) (2001) $159-170$.

44. J. Garcia de la Torre, M. L. Huertas, and B. Carrasco, Calculation of Hydrodynamic Properties of Globular Proteins from Their Atomic-Level Structure. Biophys. J. 78(2) (2000) 719-730.

45. A. Frolich, et al., From shell to cell: neutron scattering studies of biological water dynamics and coupling to activity. Faraday Discuss 141 (2009) 117-130. 